kinetic compartments and how this information is used and there is also a chapter where the ideas introduced in the book are used in worked examples of cytokinetic analysis. Only a knowledge of simple algebra is assumed and so the book should provide an invaluable guide to the analytical methods of cell and population kinetics that are in current usage.

The main fault with this book stems from its main virtue: cell kinetic analysis is not as simple as is made out. An example of this is the interpretation of one of the most commonly used techniques in cell kinetics-the Fl.M curve. The disparity between the cycle times estimated from the peaks of the FLM curve and those from labelling indices gave rise to the idea that only a fraction of the cells (the growth fraction) was active in proliferation. Perhaps the whole idea of the growth fraction needs re-analysis in view of the observation that continuous labelling with ${ }^{3} \mathrm{H}$-thymidine often labels $100 \%$ of the cells. Perhaps the growth fraction concept can be dispensed with entirely, if sufficient note is taken of the variation in the cell cycle.

This cell cycle dispersion is often treated as an inconvenient departure

\section{Popular survey of visual science}

The Miracle of Vision: The Workings and the Wonders of the Human Eye. By A. S. Freese. Pp. 181. (Harper and Row: London, 1977.) £4.50.

THE title and an introduction which includes phrases such as "the wonders of sight": "the age of aquarius": and 'the evil eye' identify this text as a short popular survey of visual and medico-visual science. Dr Freese covers in about 200 pages the relevant areas of comparative and ncuroanatomy: perception: visual physiology: the aetiology and correction of refractive errors; and much of historical and modern ophthalmology. This list could be expanded, and following the initial exuberance one could be excused for expecting to find an assortment of inaccuracies such as frequently appear in scientific works designed for the general reader. Surprisingly there are few obvious distortions induced by simplifications, and for this reason it would be unfair to single out every pedantic or minor technical grumble.

I doubt if the lay person is likely to be much interested in so many etymological notes, such as the derivations of chiasm, uveal or circadian. On adaptation it would be proper today to give equal weight to the neuroretinal as well as to the photochemical aspects of visual func- from an ideal. What is only rarely conceded is that this dispersion may be a neccssary and unavoidable consequence of the mechanics of the cycle itself. these sort of considerations have edged their way into the subconscious of some cell kineticists who now use computers and sophisticated mathematical models of the cycle in attempts to fit their data. Unfortunately, most of the data is compatible with several kinetic models; the only constant feature being the ability of the theoretical FLM curves to miss most of the experimental points. In spite of this increasing sophistication, as the authors point out. ".. to date there is not much evidence that tumour therapy has been made more effective by the use of such data".

Nevertheless, the authors have done a valuable service by explaining in simple terms the rationale behind cell kinetic analysis, although the reader should be aware that, as yet, these concepts are of more heuristic than immediate practical value.

Robert Shields

Rohert Shields works at the Imperial Cancer Research Fund Laboratories. London, UK.

tion. This book is, however, a series of popular essays, along the lines of the writings of the late A. M. Low, and it is, therefore, a pity that there are so few illustrations and no photographs. Many scientists will find the style subjective and extravagant. Who would risk describing developments in ophthalmological instruments as '. . . out of the space age's bag of electronic tricks and marvels'?

Dr Freese is subject to no such academic constraints and it is salutary to remember that it is the impersonal, objective presentation of science which is the exception in literature generally.

I have no great argument with the underlying facts, embroidered as they are for lay consumption. But my lasting reservation is whether future reality will match the expectation likely to be aroused by some sections of the work, especially those dealing with the subject of blindness. "There is even hope for the blind to "see" again', we are told, when indicating recent developments such as electrocortical implants. I accept the 'see' is given in quotes and can include sonar devices, but on this emotive aspect of vision, or lack of it, one should apply the maximum caution when presenting research developments to the public, however exciting, but on which predictions are tenuous at this early stage.

Geoffrey Ball

Geoffrey Ball is Professor of Ophthalmic Optics and Head of Department at the University of Aston in Birmingham, UK.

\section{Animal learning and human memory}

Learning and Memory. By W. A. Wickelgren. Pp. 448, (Prentice-Hall: Englewood Cliffs, New Jersey and Hemel Hempstead, UK, 1977.) $\$ 13.95$; $£ 9.75$.

THIS clear and lucid text is unusual and unfashionable; the first half presents the field of animal learning and the second that of human memory. This linkage, between areas now commonly kept quite separate, is accompanied by an emphasis on the associative quality of memory in biological systems. Such a point of view makes Pavlov's dogs reasonable neighbours for human beings memorising sentences, and restores the unity of learning lost by most recent texts. The fashionable separation is caused by experimental results suggesting quite different principles in the two cases; for example, hearing the sentence "men eat beef and ducks eat worms" produces no association of the triad "men eat worms" and the links are therefore rather different from those between the stimuli and responses of traditional conditioning. This book deals with such points by 'vertical' association of an item with a more abstract and unobserved entity, rather than with another item. Somc such device is reasonable, and indeed inevitable if language and other cognitive functions are to be evolutions of, rather than denials of, simple forms of learning.

Intelligent students will of course notice that tape-recorders or other systems regarded as "non-associative" in this book can similarly be treated as cases of vertical association, and that the emphasis on association seems then to say rather little. They may also comment that facts about animal learning mostly concern reward, punishment, and motivation, while the section on human memory scarcely mentions such points; and this rather weakens the parallelism. Their teachers will notice that, of the topics usually included in human memory, some (surprisingly few) are missing because they fit badly into the general scheme; for instance, many of the factors which alter long-term memory without affecting short-term memory.

The current fashion then has some reason behind it. But it needs challenge, and for those who want to see how far the continuity of animal and human learning can be pushed, this is an effective and engaging survey of the field.

Donald Broadbent

Donald Broadbent is an MRC staff member in the Departme'nt of Experimental Psychology at the University of Oxford, UK. 